Muséologies

Les cahiers d'études supérieures

muséologies

\title{
Le nouveau Musée historique de la ville de Strasbourg
}

\section{Monique Fuchs}

Volume 3, numéro 2, printemps 2009

URI : https://id.erudit.org/iderudit/1033570ar

DOI : https://doi.org/10.7202/1033570ar

Aller au sommaire du numéro

Éditeur(s)

Association Québécoise de Promotion des Recherches Étudiantes en Muséologie (AQPREM)

ISSN

1718-5181 (imprimé)

1929-7815 (numérique)

Découvrir la revue

Citer cet article

Fuchs, M. (2009). Le nouveau Musée historique de la ville de Strasbourg.

Muséologies, 3(2), 160-167. https://doi.org/10.7202/1033570ar d'utilisation que vous pouvez consulter en ligne.

https://apropos.erudit.org/fr/usagers/politique-dutilisation/ 
Article onze

\section{Le nouveau Musée historique}

\section{de la ville de Strasbourg}

TITULAIRE D'UNE LICENCE DALLEMAND (1976) ET DUNE MATTRISE ( LLES MONTS DES OLIVIERS SCULPTÉS SUR LE RHIN SUPÉRIEUR À LA FIN DU MOYEN ÂGEn, 1978) AINSI QUE DUNE THĖSE DE TROISIEME CYCLE EN HISTOIRE DE LART (*LA SCULPTURE EN HAUTE-ALSACEA LA FIN DU MOYEN ÁGE (1456-1521) PUBLEÉE EN 1987), MONIOUE FUCHS A ÉTÉ CHARGÉE DE LA RÉOUVERTURE DU MUSÉE DES BEAUX-ARTS DE MULLHOUSE PUIS DE LA GESTION DES MUSÉES MUNICIPAUX DE MULHOUSE DE 1982 À 1990. NOMMÉE À LA TÊTE DU CHÂTEAU DU HAUT-KOENIGSBOURG DE 1990 À 2002, ELLE Y AÉTUDIÉ LA RESTAURATION DU MONUMENT ET DÉVELOPPÉ LES ANIMATIONS. DEPUIS 2002, ELLE EST CONSERVATEUR EN CHEF DU MUSÉE HISTORIQUE DE LA VLLLE DE STRASBOURG, ROUVERT LE 1 . JUILLET 2007. [monique.fuchs@cus-strasbourgnet] 


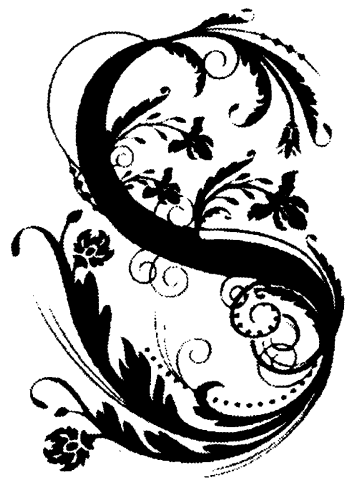

Strasbourg, ancien camp romain, est situé en Alsace au bord du Rhin, fleuve qui sépare depuis le XVII siècle l'Allemagne et la France. Bénéficiant du statut privilégié de ville libre du Saint Empire au Moyen Âge et à la Renaissance, Strasbourg sera rattaché au Royaume de France à compter de 1681. Les conflits entre la France et l'Allemagne ont marqué la région auX XIX ${ }^{\mathrm{e}}$ et XX $\mathrm{XX}^{\mathrm{e}}$ siècles (1870, 1914-1918, 1939-1945). Créé au lendemain de la Première Guerre mondiale, le Musée historique est l'un des dix musées de la ville de Strasbourg. Fermé durant 20 ans pour des raisons de sécurité liées à l'état des fondations du bâtiment, il a ouvert ses portes en juillet 2007 avec un parcours renouvelé. Les travaux associés à la rénovation du bâtiment et du musée ont été l'occasion de s'interroger sur le rôle des objets par rapport à l'histoire et notamment sur les différences entre l'objet à caractère historique et l'histoire écrite. La création du musée en 1919, au moment du retour de Strasbourg et de l'Alsace à la France après près d'un demi-siècle d'appartenance à l'Allemagne, a bien sûr influencé le choix des premières collections rassemblées au Musée historique et consacrées au Strasbourg disparu avec le bombardement de 1870. Elles reflètent aussi fidèlement les préoccupations des historiens de l'époque, qui concevaient l'histoire comme une succession de traités de paix et de déclarations de guerre et présentaient une large panoplie d'armes, d'uniformes et de petits soldats en papier.

La nouvelle conception du musée propose une sélection d'objets portant aussi bien sur les évènements que sur la vie des habitants et de ses grands hommes dans une scénographie réalisée par Laurent Marquart de la firme GSM Design (Montréal). 
Quels objets pour un musée d'histoire?

Quelle est la spécificité des objets d'un musée d'histoire au regard d'un livre? L'histoire décrite dans les documents ou racontée par les historiens est différente et complémentaire de celle qu'évoque un musée avec des objets; par exemple, il est difficile de démontrer l'esprit stratégique d'un grand général à travers le sabre qui lui a appartenu ou son portrait officiel, alors que les rapports ou les témoignages en rendent parfaitement compte. L'objet permettra un lien affectif ou dévoilera d'autres aspects de la personnalité de ce militaire. Sans vouloir dresser une typologie exhaustive des objets historiques, ces quelques lignes permettront d'entrevoir les différences entre histoire écrite et objets historiques.

La première section du Musée d'histoire de Strasbourg est consacrée à Strasbourg ville libre du Saint Empire romain germanique et au lieu où s'exerçait l'autorité de cette petite république. Une maquette du XIX $\mathrm{XIècle}^{\mathrm{e}}$ du bâtiment disparu de la " Pfalz " (c'est-à-dire l'équivalent de la mairie aujourd'hui) et un tableau prêté par le Musée des beaux-arts de Lille rendent compte de l'emplacement central, de l'architecture et d'une partie du décor de ce lieu particulièrement important pour Strasbourg. Les historiens se sont penchés sur la constitution de la ville, le musée en revanche montre que le lieu où s'exerçait l'autorité municipale était central, intégrait une ancienne chapelle et comportait une allégorie sur le thème de la Justice, exercée par le Conseil de la ville à l'époque.

Le parcours se poursuit avec des objets rappelant les trois conditions à remplir pour disposer du statut privilégié de ville "libre " : disposer de murailles et de moyens pour se défendre, avoir le privilège de frapper ses monnaies et tenir un marché ou une foire. L'activité des arquebusiers et des fondeurs de canons, les outils nécessaires à la défense, quelques monnaies strasbourgeoises et enfin les matières et les objets manufacturés importés ou exportés à Strasbourg, les mesures étalons et les métiers liés au commerce y sont présentés.

Les objets concernant les corporations sont associés à une mise en perspective d'une citation d'Érasme de Rotterdam louant, au XVI ${ }^{\mathrm{e}}$ siècle, la "République idéale" que constituait Strasbourg. L'importance des corporations qui choisissaient leurs chefs et par voix de conséquence élisaient indirectement le magistrat, c'est-à-dire le conseil municipal de l'époque, est associée à une présentation d'armoriaux et d'objets armoriés montrant les limites de cette démocratie qui ne concernait pas tous les habitants de la cité rhénane et avait tendance à coopter ses responsables. 
La portée de la mise au point de l'imprimerie au cours du XV' siècle à Strasbourg et ses conséquences sur l'humanisme et la Réforme sont perçues à travers des objets jouant le rôle de produits dérivés, illustrant les thèmes abordés dans les premières éditions d'incunables. L'évocation de l'inventeur de l'imprimerie, Gutenberg, passe par les objets commémoratifs créés en 1840 autour de la réalisation d'une statue destinée à rappeler le séjour strasbourgeois de cet homme qui révolutionna la diffusion du savoir, mais sur lequel le musée ne possède aucun objet d'époque ayant été utilisé à Strasbourg. La fin du chapitre sur la ville libre du Saint Empire romain germanique est consacrée, à travers des costumes et des portraits, au rôle des édits somptuaires sur les différentes couches de la société strasbourgeoise.

Après la présentation d'objets militaires liés à la guerre de Trente Ans, les portraits de quelques acteurs du rattachement de Strasbourg introduisent le chapitre consacré à la ville royale et aux changements induits par la capitulation de la ville. Les monopoles, exercés jusqu'alors par le magistrat, le seront désormais par le roi de France, par l'intermédiaire de l'institution du prêteur royal, tout comme d'autres fonctions exercées au nom du roi portant sur la frappe des monnaies, la fonte des canons et plus largement sur l'organisation de Strasbourg devenue ville de garnison et place forte à la frontière du royaume.

Le plan-relief de la ville remontant à 1727 permet d'évoquer l'évolution du système défensif, mais aussi l'urbanisme de Strasbourg de la Renaissance au XVIII siècle.

Quelques objets (faisselle en porcelaine de Hannong et dessins de berlines) témoignent de l'importance de certaines décisions royales sur le développement d'activités spécifiques ou leur redéploiement au-delà du Rhin.

Après une courte séquence avec peintures et objets liés à la Révolution, les peintures d'Isidore Pils (1813-1875) et des portraits rappellent que le chant patriotique français la "Marseillaise » a, en réalité, été composé à Strasbourg en 1792.

Enfin la première tranche s'achève sur l'évocation de Jean-Baptiste Kléber (1753-1800), général né à Strasbourg qui lui a dédié la place centrale de la ville. À défaut de pouvoir montrer son courage et son talent de stratège, le musée expose les reliques du grand homme, dont un nécessaire de campagne assez luxueux. La consécration du héros militaire, roturier propulsé aux plus hautes fonctions militaires grâce aux acquis de la Révolution et jouissant du statut de martyr à la suite de son assassinat, est démontrée par l'intermédiaire de l'iconographie créée à partir d'un dessin fait de son vivant et utilisé de 1800 (date de son assassinat) à 1940, en fonction des aléas politiques. 
Ce parcours muséographique s'achèvera dans les années qui viennent avec une présentation consacrée à l'évolution de Strasbourg aux XIX $\mathrm{X}^{\mathrm{e}}$ et XX $\mathrm{XX}^{\mathrm{e}}$ siècles.

Cet aperçu du parcours muséographique permet de montrer que les objets conservés au musée relèvent de différentes catégories : souvent commémoratifs (tableaux historiques, médailles), ils servent de reliques (marmite utilisée dans une circonstance historique particulière, objets ayant appartenu à un homme célèbre), illustrent de manière pragmatiquel'histoire (monnaie, mesures, sceaux, collections archéologiques) ou des échanges économiques, ou encore révèlent les valeurs politiques ou religieuses d'une époque. Le rôle d'un musée sera de se concentrer sur ce que les objets apportent à la connaissance historique plutôt que de jouer le rôle anecdotique d'illustrations ou de céder à la tentation de rédiger un livre sur des panneaux, quitte à assumer le fait de passer sous silence certains pans de l'histoire faute de pouvoir en parler avec des objets. Cela étant, l'objet se passera rarement de commentaire explicatif, d'où l'importance de la réflexion sur le public auquel est destiné le Musée d'histoire.

\section{Un Musée d'histoire pour quels publics?}

Le souci de rendre le musée accessible à un large public soulève d'autres questions.

Le Musée d'histoire de Strasbourg dispose de collections militaires (et notamment d'uniformes et de petits soldats) qui révèlent une orientation prise dans la première moitié du $\mathrm{XX}^{\mathrm{e}}$ siècle. Si ces collections spécifiques intéressant un public restreint ne sont plus exposées in extenso pour des raisons de conservation, le choix a été fait de montrer, par la variété des collections (environ 200000 pièces), l'histoire originale d'une ville frontière en se concentrant sur ce qu'elle avait de particulier.

Ce musée offre une vision généraliste, destinée à éveiller l'intérêt du public pour cette ville en incitant les visiteurs à découvrir les autres musées ou institutions culturelles strasbourgeois et ainsi à approfondir certains thèmes particuliers. Le musée ne disposant guère d'objets antérieurs au XIV siècle renvoie, par exemple pour les périodes plus anciennes, au Musée archéologique. Tout au long du parcours, des macarons circulaires signalent aux visiteurs l'existence d'autres collections spécifiques qui leur permettront d'approfondir leur envie de savoir. 
Un musée doit par ailleurs organiser la succession des objets et donc les choisir en fonction soit de thèmes, soit d'une chronologie, en privilégiant la clarté du propos. À Strasbourg le muséographe a opté pour une approche chronologique, d'une part en mettant à l'entrée de chaque section un tableau chronologique et en choisissant des couleurs tranchées selon les périodes: un fond rouge pour le Moyen Âge et la Renaissance, un fond bleu roi pour la période royale, un fond noir pour ce qui relève des périodes sombres ou du thème de la défense et à nouveau un fond rouge pour la Révolution. Sur ces fonds colorés, de grands titres aident à situer les thèmes choisis.

Un même objet pourrait se justifier à différents endroits de la visite, mais une hiérarchisation de l'intérêt de chaque pièce décide nécessairement d'un emplacement optimal. De manière concrète, il faut croiser les données techniques (poids, fragilité, problèmes de conservations) et l'intérêt commémoratif, historique, économique ou autre d'un objet pour définir son emplacement le plus favorable dans le parcours. Cela signifie qu'il est important de trouver un fil conducteur permettant au visiteur de découvrir progressivement et simplement l'histoire de la ville. Une introduction avec quelques objets empruntés au Musée archéologique évoque l'histoire de Strasbourg depuis 4500 avant Jésus-Christ; puis sont abordés les points forts de la collection, à savoir, en premier lieu, la fin du Moyen Âge, période où Strasbourg est devenue ville libre du Saint Empire. Le deuxième chapitre traite du changement profond induit en 1681 par le rattachement de Strasbourg au royaume de France. Le troisième chapitre, en cours d'élaboration, se concentrera sur les fondements d'une ville moderne, à la fois ville frontière et capitale européenne depuis 1949.

Si le parcours est découpé de manière chronologique, avec un rappel des dates importantes en début de chapitre, il propose aussi des approches thématiques, par exemple pour l'imprimerie qui est abordée depuis sa création jusqu'à nos jours ou pour l'évolution des formes des objets de la vie domestique que l'on retrouve tout au long du parcours du VIII ${ }^{e}$ siècle au XVIII ${ }^{e}$ siècle.

Le rôle du muséographe est essentiel; il permet de renforcer le propos par la mise en perspective des objets. Certains choix sont d'ordre subliminal (une paroi en verre ou des vitrines ponts pour évoquer l'importance du fleuve, ou encore l'inclinaison de cloisons pour suggérer une muraille). D'autres relèvent d'une réflexion sur la fatigabilité des visiteurs et la nécessité de varier les approches. À l'un ou l'autre endroit, l'intérêt du visiteur sera relancé par la possibilité de suivre un petit film ou spectacle associant information et humour ou émotion. 
La muséographie a recours à la vue, à l'ouie, au toucher et à des jeux pour rendre les objets accessibles à des publics différents. Les textes principaux sont courts et en trois langues, le commentaire audioguidé est ludique et bref (disponible en trois langues et à déclenchement instantané), certaines animations incitent jeunes et moins jeunes à toucher quelques objets, à en essayer d'autres ou à réfléchir, à partir d'objets ou de jeux, à l'intérêt de l'histoire pour notre présent.

L’accessibilité du musée va au-delà de la réflexion sur le mode de diffusion des connaissances auprès de publics handicapés (moteurs, visuels et autres...) pour essayer de répondre aux différents modes d'apprentissage des familles, des adultes seuls ou en groupes ainsi que des scolaires.

En conclusion, ce nouveau Musée historique, fruit d'une collaboration étroite entre l'équipe de conservation et le muséographe, espère offrir des objets dignes d'intérêt sur un parcours chargé d'émotions. La mise en perspective de ceux-ci vise à favoriser un regard critique sur le présent tout en incitant à la réflexion. 


\section{Summary}

[Translated by Michelıne Giroux-Aubin]

This article presents some of the issue of the reopening of the Strasbourg historical museum. The study of the existing collections has resulted in further analyzing both the object as it relates to history and the various roles it plays in relation to written documents. After a brief survey of the new proposed itinerary, this article evokes the museographic choices as well as the various mediation supports intended to favour the contemplation and understanding of the retained objects. The historical museum proposes 1365 objects through approximately 1700 square metres. 